\title{
Lectin binding profiles of SSEA-4 enriched, pluripotent human embryonic stem cell surfaces
}

\author{
Alison Venable ${ }^{1}$, Maisam Mitalipova ${ }^{2}$, Ian Lyons ${ }^{3}$, Karen Jones ${ }^{2}$, \\ Soojung Shin ${ }^{2}$, Michael Pierce ${ }^{1}$ and Steven Stice*2
}

\author{
Address: ${ }^{1}$ Department of Biochemistry and Molecular Biology, University of Georgia, Athens, Georgia, USA, ${ }^{2}$ Department of Animal and Dairy \\ Sciences, University of Georgia, Athens, Georgia, USA and ${ }^{3}$ BresaGen Inc, Athens, Georgia, USA \\ Email: Alison Venable - avenable@uga.edu; Maisam Mitalipova - mitalipova@hotmail.com; Ian Lyons - 17onnais@yahoo.com; \\ Karen Jones - kjones57@hotmail.com; Soojung Shin - shinso@grc.nia.nih.gov; Michael Pierce - hawkeye@uga.edu; \\ Steven Stice* - sstice@uga.edu \\ * Corresponding author
}

Published: 21 July 2005

BMC Developmental Biology 2005, 5:15 doi:10.1186/147/-2/3X-5-15

Received: 25 March 2005

Accepted: 21 July 2005

This article is available from: http://www.biomedcentral.com/I47I-2I3X/5/I5

(c) 2005 Venable et al; licensee BioMed Central Ltd.

This is an Open Access article distributed under the terms of the Creative Commons Attribution License (http://creativecommons.org/licenses/by/2.0), which permits unrestricted use, distribution, and reproduction in any medium, provided the original work is properly cited.

\begin{abstract}
Background: Pluripotent human embryonic stem cells (hESCs) have the potential to form every cell type in the body. These cells must be appropriately characterized prior to differentiation studies or when defining characteristics of the pluripotent state. Some developmentally regulated cell surface antigens identified by monoclonal antibodies in a variety of species and stem cell types have proven to be side chains of membrane glycolipids and glycoproteins. Therefore, to examine hESC surfaces for other potential pluripotent markers, we used a panel of 14 lectins, which were chosen based on their specificity for a variety of carbohydrates and carbohydrate linkages, along with stage specific embryonic antigen-4 (SSEA4), to determine binding quantitation by flow cytometry and binding localization in adherent colonies by immunocytochemistry.

Results: Enriching cells for SSEA-4 expression increased the percentage of SSEA-4 positive cells to 9899\%. Using enriched high SSEA-4-expressing hESCs, we then analyzed the binding percentages of selected lectins and found a large variation in binding percentages ranging from $4 \%$ to $99 \%$ binding. Lycopersicon (tomato)esculetum lectin (TL), Ricinus communis agglutinin (RCA), and Concanavalin A (Con A) bound to SSEA-4 positive regions of hESCs and with similar binding percentages as SSEA-4. In contrast, we found Dolichos biflorus agglutinin (DBA) and Lotus tetragonolobus lectin (LTL) did not bind to $\mathrm{hESC}$ s while Phaseolus vulgaris leuco-agglutinin (PHA-L), Vicia villosa agglutinin (VVA), Ulex europaeus agglutinin (UEA), Phaseolus vulgaris erythro-agglutinin (PHA-E), and Maackia amurensis agglutinin (MAA) bound partially to hESCs. These binding percentages correlated well with immunocytochemistry results.

Conclusion: Our results provide information about types of carbohydrates and carbohydrate linkages found on pluripotent hESC surfaces. We propose that TL, RCA and Con A may be used as markers that are associated with the pluripotent state of $\mathrm{hESCs}$ because binding percentages and binding localization of these lectins are similar to those of SSEA-4. Non-binding lectins, DBA and LTL, may identify differentiated cell types; however, we did not find these lectins to bind to pluripotent SSEA-4 positive hESCs. This work represents a fundamental base to systematically classify pluripotent $\mathrm{hESCs}$, and in future studies these lectins may be used to distinguish differentiated hESC types based on glycan presentation that accompanies differentiation.
\end{abstract}




\section{Background}

Ever since the isolation of human embryonic stem cells (hESCs) in 1998 [1], the implications for their use in a number of disease therapies have been highly regarded. Additionally, these cells also find value as a model to study basic human development. However, in all aspects of ESC research, hESCs must first be appropriately defined or characterized. One way to characterize hESCs is to utilize the large number of glycoproteins and carbohydrates existing on the cell surface as a way to delineate pluripotent or differentiated cell types.

The most common hESC surface pluripotency markers are the stage specific embryonic antigens -3 and -4 (SSEA-3, 4) and tumor rejection antigens-1-60 and -1-81 (TRA-1$60,-1-81)$. SSEA-3 and -4 are globoseries cell surface glycoproteins that were first used to delineate embryological changes in the developing mouse embryo [2,3]. Both of these antigens were found to recognize sequential regions of a mouse ganglioside epitope, with SSEA-4 (MC813-70 antibody) recognizing the terminal portion of the sequence and SSEA-3 (MC613) recognizing the internal region of he sequence. Thus, two antibodies were used to define this unique embryonic antigen. In mouse embryonic stem cells (mESCs), SSEA-3 and -4 are expressed on the 2-8 cell and morula stages of preimplantation embryos and are also found on unfertilized oocytes; however, there is a loss of expression in the inner cell mass (ICM) of mESCs [2,3]. Yet in hESCs, there is no expression of SSEA-3 or -4 at the $2-8$ cell or morula stage; however, these are expressed on the ICM of human blastocysts and on isolated hESCs [4]. It has been well documented that these cell surface carbohydrates change both with development and differentiation in vitro $[5,6]$, but there may be other undiscovered developmentally regulated cell surface carbohydrates.

The hematopoetic field has numerous cell surface antigens which have been identified that define various bone marrow and blood stem cells, such as CD4, CD8, CD34, CD38, CD44, CD45, c-kit, Mac-1, Muc -18, Lin, Sca-1, and Thy-1 [7-11] and there has been some progress in identifying other hESC surface markers that can be used both for defining the pluripotent state and for cell sorting and cell identification. Cell surface markers such as CD9 and CD24 are presented on pluripotent hESC surfaces, while gene expression analysis indicates such genetic markers as REX1 [12], Cripto/TDGF1 [12], OCT-4 [13], DNMT3B [13], LIN28 [13], Nanog [14], and others that can denote pluripotency in hESCs [13]. Since carbohydrate antigens on hESC surfaces could provide further potential markers defining the pluripotent state, we targeted glycosylation patterns on the cell surface of SSEA-4 enriched hESCs using lectins to bind to these glycans. Lectins, carbohydrate binding proteins that recognize diverse sugar struc-

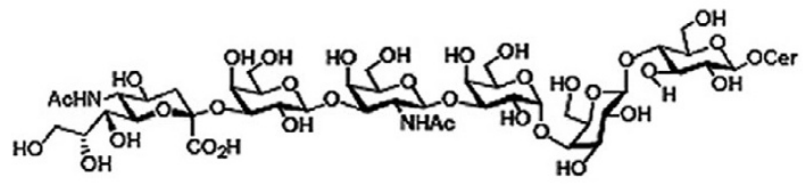

Figure I

Structure of Stage Specific Embryonic Antigen 4

(SSEA4). SSEA-4, one of the most commonly used hESC surface pluripotency markers, is a globoseries glycolipid that is characteristically downregulated upon hESC differentiation.

tures, have been extensively used to identify and characterize cell surface glycosylation patterns. For example, lectins have been used to investigate metastatic processes in many types of cancer [15-21] and to identify cell types based on presentation of specific cell surface carbohydrates [22-27]. Carbohydrate analysis using lectins has also led to the delineation of embryologic developmental stages in some species. For example, many developmentally regulated glycans identified as lectin receptors on mESCs are displayed on cell surfaces at the preimplantation and implantation stages of development. These include Concanavalin A (Con A) defined by the hapten methylmannoside [28], Peanut agglutinin (PNA) from Arachis hypogaea defined by the hapten galactose [29], Wheat Germ Agglutinin (WGA) from Triticum vulgaris defined by hapten N-acetylglucosamine [30], Dolichos biflorus agglutinin (DBA) defined by the hapten N-acetylgalactosamine [31], and Ricinus communis agglutinin (RCA) defined by the hapten galactose or lactose [32]. These results suggest that glycans may be involved in cellcell interactions serving a specific developmental function and also indicate that they can be used as markers to define these stages of mouse embryogenesis.

In this study, we analyzed the pluripotent state of two NIH approved hESC lines, BG01 and BG02. These cell lines have previously been characterized for positive staining of the pluripotency markers TRA-1-60 and -1-81, OCT-4, alkaline phosphatase, and SSEA-3 and -4 [33]. Lectin binding percentages on SSEA- 4 enriched hESC surfaces were determined by flow cytometry using a panel of 14 lectins and SSEA-4, which enabled us to probe the surface of pluripotent hESCs for a variety of carbohydrates and carbohydrate linkages. Rosler et al showed that in long-term culture, hESCs maintained pluripotency as determined by measurement of a variety of characteristic markers, including SSEA-4, TRA-1-60 and TRA-1-81 [34]. Moreover, there was a high correlation between expression of SSEA- 4 and TRA-1-60 and -1-81, and the presence of these markers correlated with undifferentiated 


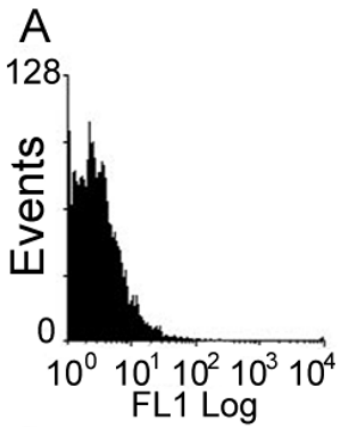

Figure 2

SSEA-4 expression in enriched hESCs determined by flow cytometry. The histogram plots of unstained, control hESCs (2A) and cells stained with SSEA-4 antibody after enrichment using magnetic bead sorting at day 0 (2B).

morphology. Thus, we defined pluripotent hESCs as those populations with $98-99 \%$ expression of SSEA-4, a developmentally regulated cell surface pluripotency marker that is frequently used to delineate pluripotent hESCs $[1,4,5,35]$. We were able to obtain this high level of SSEA4 expression by using magnetic bead sorting to select SSEA-4 expressing cells. The SSEA-4 antibody (MC 81370) specifically recognizes a carbohydrate chain (Figure 1); thus, we analyzed a panel of lectins that are likely to be presented on hESC surfaces in order to identify other potential markers for pluripotent hESCs. Immunocytochemistry using SSEA-4 and each of the 14 lectins was analyzed to determine localization of lectin binding within adherent hESC colonies and to validate binding specificity of each lectin using appropriate competitive sugars.

We found that a variety of lectins binding unique carbohydrate moieties had distinct binding patterns. Our findings demonstrate that there are many surface carbohydrate antigens that could be exploited to further characterize the pluripotent state of hESCs, and these lectins may provide a source of unique markers with which to characterize subpopulations that exist in colonies of adherent hESCs.

\section{Results}

\section{Carbohydrate analysis using flow cytometry}

HESCs were trypsin passaged and selected for SSEA-4. It is important to note that manual passaging would not be feasible for flow cytometry studies, since atleast 250,000 single cells are needed per treatment for flow cytometry analysis. After SSEA-4 enrichment using magnetic bead sorting, $99.3 \pm 0.31 \%$ of hESCs expressed SSEA-4 (Figure 2 ). To determine the presence and the percent binding of

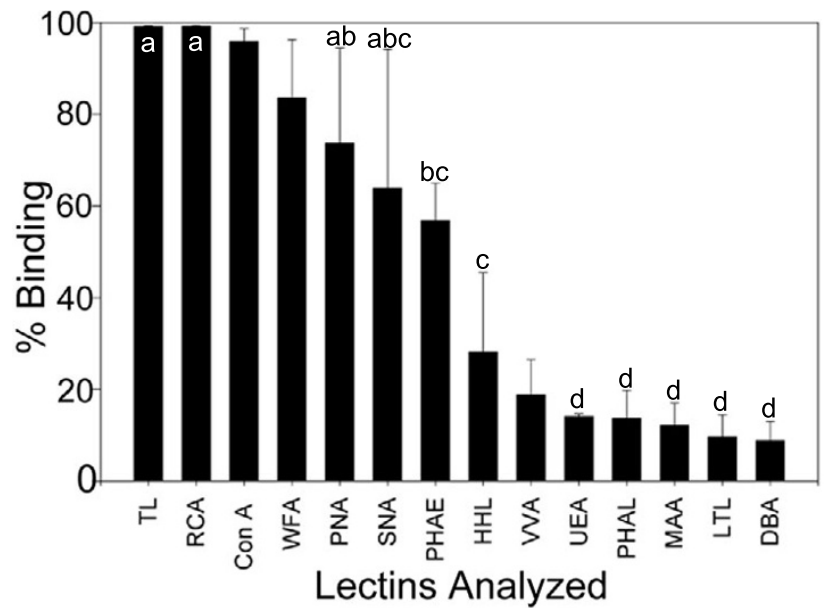

Figure 3

Quantitation of lectin binding on pluripotent hESC surfaces with I 4 different lectins. The percent of cells with specific carbohydrate expression as determined by flow cytometry using 14 different lectins. The data are means +/SD of 3 independent assays of BGOI and BG02 hESC lines. hESCs from each line were stained with one of 14 lectins and SSEA-4 immediately following enrichment for SSEA-4 expression. abcd: Means with different letters are significantly different, $p<0.05$.

the 14 chosen lectins on pluripotent hESC surfaces, both karyotypically normal hESC lines (BG01 and BG02) were analyzed using flow cytometry with the panel of lectins and the pluripotency marker, SSEA-4. A broad range of binding percentages was observed (Figure 3 ). The highest binding percentages were detected using TL, RCA, ConA, WFA, and SNA. Binding percentages for TL, RCA, and ConA were similar to that of SSEA-4, ranging from 98$99 \%$ of enriched hESCs. Figure 4B shows a shifted histogram plot of TL as a representative of these high percentage binding lectins. WFA, PNA and SNA were also found to bind to over $60 \%$ of hESCs.

Two lectins, DBA and LTL, did not bind to hESCs. Analysis of DBA and LTL histograms showed no peak shifts using these lectins (Figure 4C), and analysis of flow cytometry plots also did not reveal any peak shifts (supplementary data).

Some lectins were found to partially bind to hESC colonies. These lectins revealed two distinct subpopulations of cells that included a SSEA4+/lectin- population and a SSEA4+/lectin+ population. Figure 4D shows a representative histogram plot of MAA which shows a large population of cells that overlap the unstained control cells; however, the arrow indicates another smaller population 

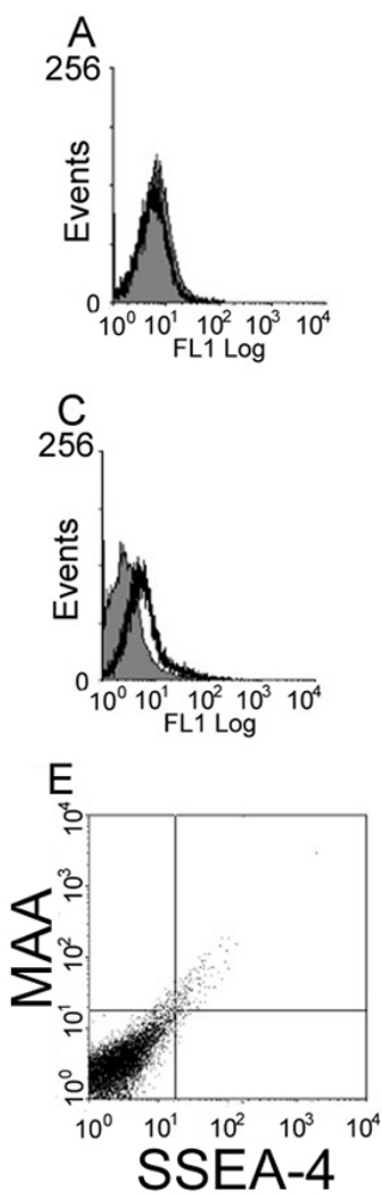
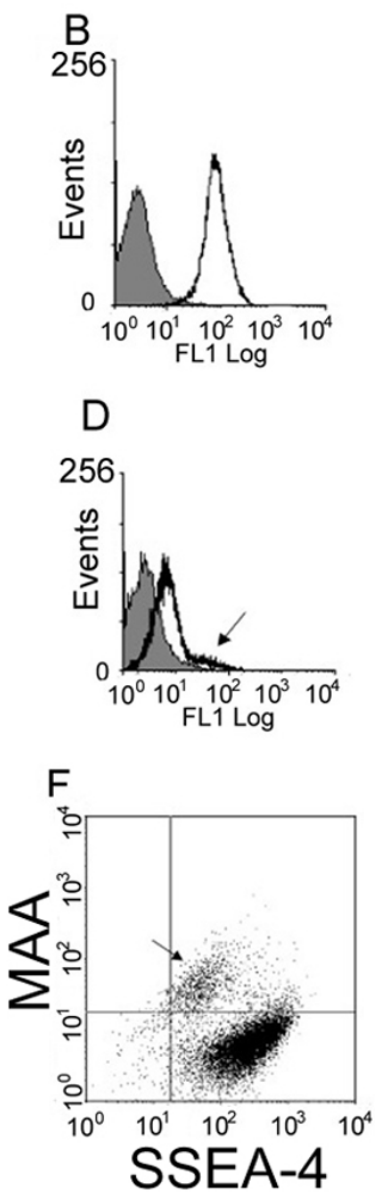

Figure 4

Flow cytometry histograms of lectin binding in unstained and stained HESCs. A-D shows histograms of SSEA-4 binding and representative lectins that were used in this study. To validate that double staining can be performed without signal interference, we determined that SSEA-4 expression was not found in the FLI channel. Figure 4A shows a histogram plot with the overlay image of SSEA4 (black tracing) matching the histogram plot of unstained cells (grey fill). Figure 4B shows a positive peak shift in the histogram overlay with Tomato lectin (TL) in black tracing and unstained cells (grey fill). Figure $4 \mathrm{C}$ shows lack of Lotus tetragonolobus lectin (LTL) (black tracing) binding in the histogram overlay with unstained cells (grey fill). Figure 4D shows a histogram overlay with MAA (black tracing) binding and shows two peaks -one representing a large population that overlays unstained cells and a smaller population denoted by arrow that shows a smaller population of MAA+/SSEA4+ cells. Figure 4E shows plots of unstained hESCs, and Figure 4F shows subpopulations of cells characterized as SSEA-4+/ MAA- or as SSEA-4+/MAA+ cells (arrow).

of cells that show a peak shift representing positive MAA binding. Figure $4 \mathrm{E}$ shows the comparison of unstained control cells to the shifted plot of MAA (Figure 4F) in which there are two distinct populations of cells present: a SSEA-4+/lectin- population containing the majority of cells, and a smaller but still distinct SSEA-4+/lectin+ population indicated by the arrow. Lectins that bound in this way included PHA-E, VVA, UEA, PHA-L, and MAA, and these exhibited a large binding range varying from $58 \%$ to $13 \%$ binding of enriched SSEA-4 positive cells (Figure 3 ).

\section{Carbohydrate analysis using immunocytochemistry}

Both hESC lines, BG01 and BG02, were analyzed by immunocytochemistry to determine localization of carbohydrates and to determine whether particular staining patterns were present in adherent colonies maintained in culture. Cells were either passaged manually or by trypsinization and we did not observe obvious differences in hESC colony staining patterns between these passage methods. Immunocytochemistry results of all 14 lectins supported our flow cytometry analysis. Five lectins (TL, RCA, ConA, WFA, SNA, and HHL) bound throughout the colonies without any localized patterns of binding. TL, RCA, ConA, and WFA appeared to bind to cells that also expressed SSEA-4. Figure 5A-C shows a hESC colony that represents uniform lectin binding. RCA binding (Figure $5 \mathrm{~A}$ ) is shown throughout a colony that is SSEA-4 positive (Figure $5 \mathrm{~B}$ ) and is also stained with DAPI nuclear stain (Figure 5C).

We also observed interesting patterns of carbohydrate expression for the lectins that showed two subpopulations of cells when analyzed using flow cytomtery. VVA bound to distinct regions of a SSEA-4 positive colony (Figure 5D-F), in contrast to the more uniform binding described above. In Figure 5D the arrow shows a region where there is no VVA binding, but this region is SSEA- 4 positive as shown in Figure 5E. The DAPI nuclear stain of this same colony is shown in Figure 5F. Figures $5 \mathrm{G} \& \mathrm{H}$ show PHA-E binding in different colonies. In Figure 5G there are two adjacent colonies with one colony showing strong binding of SSEA- 4 antibody (green) and little PHAE binding (red). The adjacent colony shows binding of PHA-E but a lack of SSEA-4 antibody binding. In Figure $5 \mathrm{H}$ there is one colony that shows a streak of stacked cells as determined by the high expression of DAPI staining in the middle of the colony (depicted by arrow). These cells are beginning to lose SSEA-4 antibody binding (green) but have strong PHA-E binding (red). The rest of the colony adjacent to this streak of cells is uniformly positive for SSEA-4 but lacks PHA-E binding.

Using immunocytochemistry we also demonstrated that DBA and LTL did not bind to hESC colonies. Figure 5I shows a hESC colony which is SSEA-4 positive (green) 

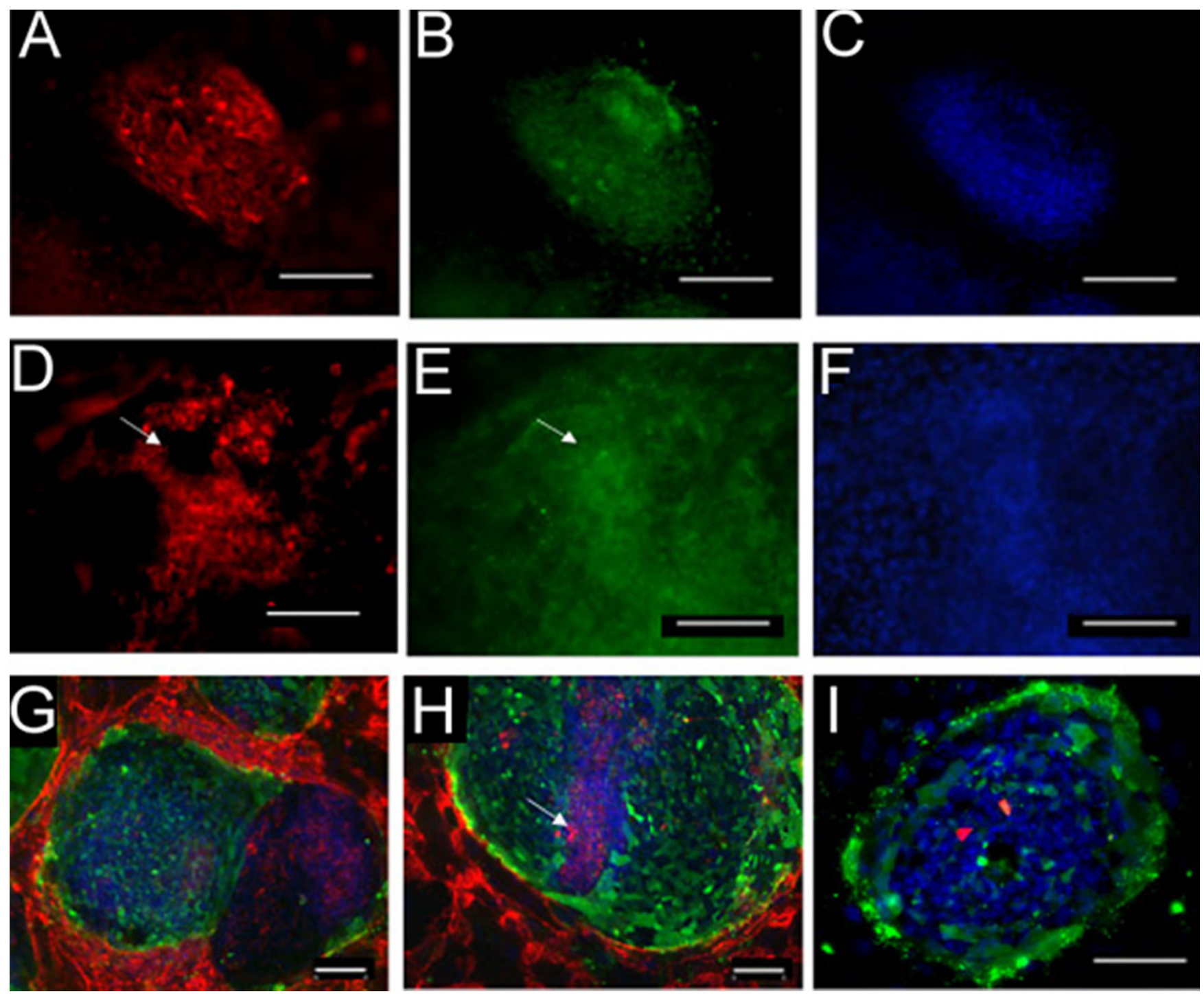

\section{Figure 5}

Carbohydrate expression as determined by lectin binding using immunocytochemistry. Figure 5A-C shows a hESC colony that represents uniform lectin binding. Ricinus Communis agglutinin (RCA) binding in red (5A) is shown throughout this SSEA-4 positive colony in green (5B). The DAPI nuclear stain image (blue) is also shown (5C). Other lectins showed partial binding patterns, such as Vicia Villosa agglutinin (VVA) binding (red), which is shown in a hESC colony (5D) that has uniform SSEA-4 antibody binding (green) (5E). Arrows denote distinct SSEA-4 positive regions lacking VVA binding. DAPI nuclear staining (blue) is also shown (5F). PHA-E binding is shown in two separate images in Figure $5 \mathrm{G}-\mathrm{H}$. In $5 \mathrm{G}$ there are two adjacent colonies, one that expresses strong binding of SSEA-4 antibody (green) and weak to no binding of PHA-E (red), and an adjacent colony showing binding of PHA-E without SSEA-4 antibody binding. (DAPI nuclear staining in blue). 5H, shows another colony with a streak of stacked cells (as determined by high DAPI expression, see arrow) in the middle of the colony that are beginning to lose SSEA-4 expression (green), but have strong PHA-E binding (red). However, the rest of the colony adjacent to this streak of cells is uniformly positive for SSEA-4 but is lacking PHA-E binding. 5I shows lack of DBA binding and presence of SSEA-4 and DAPI staining. Images and scale bars: 5A-G) 20× magnification, $100 \mu \mathrm{m}$. H-I) I0× magnification, $100 \mu \mathrm{m}$.

and is stained with DAPI nuclear stain (blue) but shows only debris of the DBA lectin (red) with no regions of DBA binding.

\section{Validation of lectin binding}

We used complementary competitive sugars that are known to block lectin binding (listed in Table 1) to 
Table I: Comparison of the specificity for monosaccharides and oligosaccharides of a panel of I 4 biotinylated lectins used in immunocytochemistry and flow cytometry.

\begin{tabular}{lll}
\hline \multicolumn{1}{c}{ Lectin Origin } & Monosaccharide Specificity & \multicolumn{1}{c}{ Inhibitor } \\
\hline Concanavalin A (Con A) & Man or Glc & $200 \mathrm{mM} \alpha$-methylmannoside or $\alpha$-methyl glucoside \\
Phaseolus vulgaris erythro-agglutinin (PHA-E) & Gal & galactose \\
Phaseolus vulgaris leuco-agglutinin (PHA-L) & Gal & galactose \\
Sambucus nigra agglutinin (SNA) & Sialic Acid & $500 \mathrm{mM}$ lactose in acetic acid \\
Arachis hypogea peanut (PNA) & Gal & $200 \mathrm{mM}$ galactose \\
Vicia villosa agglutinin (VVA) & GalNAc & $200 \mathrm{mM}$ N-acetylgalactosamine \\
Maackia amurensis (MAA) & Gal & $200 \mathrm{mM}$ lactose \\
Ricinus communis agglutinin (RCA) & Gal & $200 \mathrm{mM}$ galactose or lactose \\
Wisteria floribunda agglutinin (WFA) & GalNAc & $200 \mathrm{mM}$-acetylgalactosamine \\
Ulex europaeus agglutinin (UEA) & Fuc & $50-100 \mathrm{mM}$ L-fucose \\
Lotus tetragonolobus lectin (LTL) & Fuc & $50-100 \mathrm{mM}$ L-fucose \\
Dolichos biflorus agglutinin (DBA) & GalNAc & $200 \mathrm{mM}$-acetylgalactosamine \\
Hippeastrum hybrid lectin (HHL) & Man & $100 \mathrm{mM}$ mannose \\
Lycopersicon esculetum tomato (TL) & GlcNAc & Chitin Hydrolysate
\end{tabular}
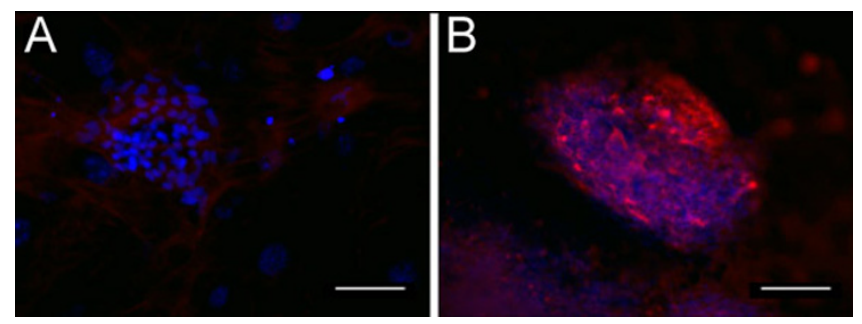

Figure 6

Addition of competitive sugar inhibitor blocks lectin expression as determined by immunocytochemistry. Addition of $200 \mathrm{mM}$ galactose can block RCA binding in a hESC colony as shown by lack of RCA binding (6A), but does not affect DAPI stain (blue). However, in the absence of galactose, uniform binding of RCA (red) was detected 6(B). 6A-B) $20 \times$ magnification, $100 \mu \mathrm{m}$

validate lectin binding specificity. The concentrations listed in Table 1 were all found to inhibit each lectin's binding as detected by immunocytochemistry. As a representative of competitive sugar inhibition, Figure 6 shows blocking of RCA binding by addition of $200 \mathrm{mM}$ galactose (Figure 6A). In the absence of galactose, RCA binding is evident (red, Figure 6B).

\section{Discussion}

This study is the first of which we are aware to use lectins to probe the surface of hESCs in order to analyze carbohydrate presentation. To quantitate lectin binding percentages on pluripotent hESC surfaces using flow cytometry, we first enriched our colonies for SSEA-4 expression. Using enriched pluripotent hESCs, we found a large bind- ing percentage range for this chosen panel of 14 lectins. Some lectins bound throughout the colonies of pluripotent hESCs, and the carbohydrate moieties represented by this group include alpha-linked mannose (Con A), galactosyl $(\beta 1-3) \quad \mathrm{N}$-acetylgalactosamine (PNA), terminal galactose and N-acetylgalactosamine (RCA), GalNAc $\beta 4$ Gal (WFA), N-acetylglucosamine (TL) and sialic acid alpha 2,6 GalNAc (SNA) and alpha-linked mannose residues only (HHL). These results indicate that there is high expression of these carbohydrates with these particular linkages on pluripotent hESC surfaces. As ConA can bind both mannose and glucose moieties, we see greater lectin binding of ConA as opposed to HHL, which specifically binds only mannose residues. These lectins may be additional markers to denote pluripotent hESCs. Similarly, WFA, PNA and SNA were found to bind to over $60 \%$ of hESCs, suggesting that these lectins could be indicative of a pluripotent phenotype as well; however, they do not have binding percentages that correlate with SSEA- 4 expression.

We also found that some lectins showed interesting partial binding patterns. Two distinct populations of cells were observed as determined by flow cytometry plot analysis and confirmed by immunocytochemistry using these lectins and SSEA-4 antibody. These hESC populations were identified as either SSEA-4+/lectin+ or SSEA-4+/lectin- regions. Lectins showing this binding pattern included PHA-L, VVA (see Figure 5D-F), UEA, PHA-E (Figure 5G-H), and MAA. Table 1 shows their carbohydrate binding specificities.

As shown by immunocytochemistry of the representative lectin, PHA-E, it appeared that some areas that were beginning to lose expression of SSEA-4, show binding of 
the lectin (Figure 5G-H). A decrease of SSEA-4 expression can occur even under the most stringent culturing conditions and in this research, is important because it underscores the need for enriching for desired populations of cells prior to experimentation and also supports the hypothesis that cell-cell interactions are important in carbohydrate presentation. As clusters of cells lose SSEA-4 expression, and therefore differentiate, new carbohydrates are presented on the cell surface. Furthermore, these differentiated cells could be indicative of progenitor subpopulations existing in the colonies. For example, another lectin in our study that was found to bind partially to colonies is UEA, which binds fucose moieties and has been determined to be a marker for endothelial cells [16]. The role these partially presented carbohydrates play was not investigated in this study, but others have suggested that certain carbohydrates may serve a developmental function. For example, Panin and colleagues have identified O-fucose on epidermal growth factor-like repeats of Notch, and elongation of O-fucose has been implicated in the modulation of Notch signaling by Fringe $[36,37]$. Notch receptors and associated proteins are important in a number of signaling pathways that direct cell fate decisions, proliferation and apoptosis.

We also observed that two lectins, DBA and LTL, did not bind hESCs. DBA binds primarily to $\alpha$-linked $\mathrm{N}$-acetylgalactosamine and LTL binds fucose moieties that are $\alpha$ linked to GlcNAc. These non-binding lectins suggest that these particular carbohydrates and their associated linkages are either not present on pluripotent hESC surfaces or if they are present, they have been modified and therefore are not susceptible to lectin binding. Interestingly, however, PNA and WFA, which bind to a high percentage of hESCs, bind to $\beta$-linked $\mathrm{N}$-acetylgalactosamine. Thus, it appears that the linkage of this carbohydrate is important. Similarly, UEA binds to about $20 \%$ of our cells and recognizes fucose moities like LTL. However, the linkages that these lectins recognize are different and cause different binding percent outcomes, further suggesting that the linkage of these carbohydrates are important.

Developmentally regulated cell surface antigens have been identified by monoclonal antibodies in a variety of species and stem cell types. Many of these antigens have proven to be side chains of membrane glycolipids and glycoproteins. For example, the SB10 antigen found on human mesenchymal stem cells was shown to be the activated leukocyte-cell adhesion molecule (ALCAM) that possesses N-linked oligosaccharide side chains [38]. Muramatsu and colleagues discovered a protein called embigin that has a developmentally regulated carbohydrate chain that is lost upon mouse embryogenesis [39]. Furthermore, other researchers showed that the monoclonal antibody GCTM-2, which binds to human embryonic carcinoma cells and primate ES cells, recognizes an epitope on a keratan sulfate proteoglycan $[40,41]$. These studies have shown not only that carbohydrates on cell surfaces change with embryological development but also that they can be used to identify different types of stem cells. In addition, there are a number of carbohydrate epitopes that have been found on the mammalian preimplantation embryo. Ofcourse, the tumor rejection antigens and stage specific antigens have been well characterized on human embryos [4], but there are many others such as the TEC antigens (TEC1- TEC4), LeX, LeY, CD46, CD55, and CD59. The TEC antigens have been shown to recognize either the carbohydrate moiety of embryoglycan or a developmentally regulated protein epitope on both mouse and bovine preimplantation embryos [42]. LeX [Galbeta1-4(Fucalpha1-3)GlcNAc] is first detected on the blastomeres of the 8-cell stage of the mouse embryo and LeY [Fucalpha1-2Galbeta14(Fucalpha1-3)GlcNAc] is highly expressed on the surface of the mouse blastocyst $[43,44]$. Also, studies of human preimplantation embryos show that the glycosyl phosphatidylinositol anchored proteins CD46, CD55 and CD59 are expressed on oocytes and plasma membrane [45]. In this study, we analyzed glycans on the cell surface of hESCs using a panel of 14 lectins and SSEA- 4 antibody and found that pluripotent hESC surfaces have a large variety of surface carbohydrates that can be targeted for marker sources.

\section{Conclusion}

This work represents a fundamental base to systematically classify pluripotent hESCs, and in future studies these lectins may be used to distinguish differentiated hESC types based on carbohydrate presentation that accompanies differentiation. It will be important to determine the potential of these lectin positive cell types and to identify the cell surface glycoproteins or glycolipids to which these lectins bind. Because there are many different enzymes that add or remove carbohydrate structures on cell surfaces, it will also be interesting to investigate the function of some of these glycosyltransferases in both pluripotent and differentiated hESCs.

\section{Methods \\ Cell culture and passaging}

$\mathrm{NIH}$ approved hESC lines BG01 and BG02 with normal karyotypes were obtained from BresaGen, Inc. [33]. Cells were grown in DMEM/F12 supplemented with 15\% FCS (HyClone), 5\% knockout serum replacer, $1 \times$ non-essential amino acids, $20 \mathrm{mM} \mathrm{L-} \mathrm{Glutamine,} 0.5 \mathrm{U} / \mathrm{ml}$ penicillin, $0.5 \mathrm{U} / \mathrm{ml}$ streptomycin, $4 \mathrm{ng} / \mathrm{ml}$ FGF-2 (Sigma), (all from Gibco Invitrogen unless otherwise labeled). Human ESCs were maintained on mitotically inactivated primary mouse embryonic fibroblasts (MEF) feeder layers for routine maintenance. Cells were grown in 
$100 \mathrm{~cm}$ tissue culture treated dishes (Falcon). Cells were passaged every 3 days using either a pretreatment with 10 $\mathrm{mg} / \mathrm{ml}$ collagenase for 2 minutes followed by $0.05 \%$ trypsin for 1 minute or manual dissection with a fire pulled Pasteur pipette [33]. Immunocytochemistry was performed on routinely maintained adherent hESC colonies, and flow cytometry was performed using routinely maintained hESC colonies that were first enriched for SSEA-4.

\section{Enrichment of SSEA-4 positive cells}

Cultures of hESCs were grown in $100 \mathrm{~cm}$ dishes and trypsin passaged into single cell suspensions as described above. Cells were incubated on ice for 15 minutes in 1:10 dilution of SSEA-4 (MC 813-70, Developmental Studies Hybridoma Bank [DSHA]; Iowa City; in stain buffer (SB). SB consisted of $0.5 \mathrm{ml} 0.5 \mathrm{U} / \mathrm{ml}$ penicillin, $0.5 \mathrm{U} / \mathrm{ml}$ streptomycin, $0.5 \mathrm{ml}$ of $100 \mathrm{mM}$ EDTA, $46.5 \mathrm{ml}$ phosphate buffered saline (PBS) and $2.5 \mathrm{ml}$ fetal bovine serum (FBS). After incubation $10 \mathrm{ml} \mathrm{SB}$ was added and cells were resuspended and centrifuged at $3000 \times \mathrm{g}$ for 5 minutes. Supernatant was removed, leaving the cell pellet intact, and cells were washed again in SB and resuspended. After centrifugation, a 1:4 dilution of secondary anti-mouse IgG in stain buffer was added to the cell pellet, and the resuspended pellet was incubated on ice for 25 minutes. After incubation $10 \mathrm{ml}$ SB was added to wash the cell pellet, followed by centrifugation $(3000 \times \mathrm{g})$ for 5 minutes. This process was repeated two more times in $5 \mathrm{ml} \mathrm{SB}$. Cells were finally resuspended in $500 \mu \mathrm{l}$ of SB before being applied to a pre-washed magnetic bead column. The flow through from the column was collected and saved for counting, and the retained eluate was collected separately. Both flow through and eluate were brought up to $5 \mathrm{ml}$ in SB after collection and counted [46]. Cells were then subjected to flow cytometry. Flow cytometry results presented here are from hESCs that were used immediately after the enrichment procedure.

\section{Flow cytometry}

Cell surface antigen and carbohydrate expression of SSEA$4+$ enriched hESCs was assessed by indirect immunofluorescence detected by flow cytometry to provide a quantitative binding percentage of both SSEA-4 and lectin. Human ESCs were harvested into single cell suspensions using trypsinization as described above. Cells were enriched for SSEA-4 as described above. Then, cells were fixed in $2 \%$ paraformaldehyde in $1 \times$ PBS. After blocking in $1 \%$ bovine serum albumin (BSA) for 30 minutes, cells were placed in sterile conical tubes in aliquots of 500,000 cells each and double stained with one of the 14 lectins at $5 \mu \mathrm{g} / \mathrm{ml}$ and SSEA-4 in a 1:100 dilution. Cells were washed 3 times with PBS and then stained with secondary antibodies that included streptavidin -allophycocyanin (1:250, BD Biosciences; Franklin Lakes, NJ;) for recognition of biotinylated lectins and antigoat Mouse IgG conjugated Alexa 488; (1:2000) for recognition of SSEA-4. These secondary antibodies were chosen so that there would not be overlap in the emission/excitation wavelengths and so that double staining could be performed. Unstained, enriched hESCs and enriched hESCs stained with secondary antibodies alone were used as controls.

Table 1 shows the chosen tested lectins, their commonly abbreviated name, and the specificity of these lectins for their respective monosaccharides to act as a quick reference guide and the concentration of hapten, or competitive sugar, that was used to verify specificity of each lectin for its carbohydrate. Specificity of each lectin has previously been described in detail by Cummings and colleagues [47]. Cytometry was performed using a Beckman Coulter Cytomics FC 500 Flow Cytometer. Data analysis was performed using the RXP Analysis Software by Beckman Coulter and Windows Multi Document Interface for Flow Cytometry (WinMDI 2.8). Three independent assays were carried out using both BG01 and BG02 hESC lines.

\section{Immunocytochemistry}

Immunocytochemistry was used to analyze the localization of cell surface carbohydrate expression and SSEA-4 on routinely maintained adherent cultures of hESCs. Human ESCs were harvested from $100 \mathrm{~cm}^{2}$ dishes by either trypsin/collagenase dissociation or manual dissociation and fixed in $4 \%$ paraformaldehyde (Fisher Scientific) in $1 \times$ PBS (Gibco) for 30 minutes. After blocking in $1 \%$ BSA solution for 30 minutes, cells were equally divided into 4-well chamber slides which had previously been plated with mitotically inactivated MEFs. Cells were then double stained with one of 14 biotinylated lectins (all lectins obtained from Vector Laboratories; Burlingame, CA; $10 \mu \mathrm{g} / \mathrm{ml}$ ) and SSEA 4 (MC 813-70, Developmental Studies Hybridoma Bank [DSHA]; Iowa City; $1: 100$ ) for 20 minutes at $37^{\circ} \mathrm{C}$, followed by 3 washes in PBS. Secondary antibodies included streptavidin conjugated Alexafluor 594 (Molecular Probes; Eugene, OR; 1:250 dilution) and antigoat Mouse IgG conjugated Alexa 488 (Molecular Probes; 1:2000 dilution). During staining procedure, cells were kept at $37^{\circ} \mathrm{C}$. Cells were washed three times after secondary antibody incubation for 5 minutes in PBS. Cells were then post-stained with $5 \mathrm{ng} / \mathrm{ml}$ DAPI (Sigma) to detect cell nuclei and washed overnight in PBS. Controls of unstained cells were obtained by incubation with secondary antibodies alone. All slides were mounted and visualized using a Nikon TS100 inverted microscope. Individual color channels were captured separately and merged in Adobe Photoshop.

\section{Abbreviations used}

human embryonic stem cells (hESCs) 
stage specific embryonic antigen-4 (SSEA-4)

inner cell mass (ICM)

$\mathrm{N}$-acetylgalactosamine (GalNAc)

Fibroblast growth factor-2 (FGF-2)

mouse embryonic fibroblasts (MEF)

stain buffer (SB)

phosphate buffered saline (PBS)

fetal bovine serum (FBS)

bovine serum albumin (BSA)

Concanavalin A (Con A)

Phaseolus vulgaris erythro-agglutinin (PHA-E)

Phaseolus vulgaris leuco-agglutinin (PHA-L)

Sambucus nigra agglutinin (SNA)

Arachis hypogea peanut (PNA)

Vicia villosa agglutinin (VVA)

Maackia amurensis (MAA)

Ricinus communis agglutinin (RCA)

Wisteria floribunda agglutinin (WFA)

Ulex europaeus agglutinin (UEA)

Lotus tetragonolobus lectin (LTL)

Dolichos biflorus agglutinin (DBA)

Hippeastrum hybrid lectin (HHL)

Lycopersicon esculetum tomato (TL)

\section{Authors' contributions}

$\mathrm{AV}$ carried out the maintenance and culturing of the hESCs, flow cytometry and immunocyotochemical studies and drafted the manuscript. MM helped in maintenance of hESCs, immunocytochemistry, and aided in manuscript review. KJ participated in flow cytometry set up. SS, IL, and MP participated in the design of the study. SS participated in its design and coordination and helped to draft the manuscript. All authors read and approved the final manuscript.

\section{Links}

Developmental Studies Hybridoma Bank

http://www.uiowa.edu/ dshbwww

BD Biosciences

http://www.bdbiosciences.com

Vector Laboratories

http://vectorlabs.com

Molecular Probes

http://www.probes.com

\section{Additional material}

\section{Additional File 1}

Histogram plots of other lectins tested. Figure S1 shows peak shifts of RCA (A), PNA (B), ConA (C), WFA (D), SNA (E), and PHA-L (F) binding in black tracing, and unstained cells in grey fill. Click here for file

[http://www.biomedcentral.com/content/supplementary/1471213X-5-15-S1.PDF]

\section{Additional File 2}

Flow cytometry plots of other lectins tested. Figure S2 shows plots of some lectins that have 2 populations of cells such as PHA-L (A), UEA (B), and VVA (C). The plot of CON A (D) indicates only one population of cells showing a positive shift for lectin and SSEA-4 antibody binding, while the plot of DBA (E) and LTL (F) also show only one population of cells with no shift in lectin binding, but a positive shift in the SSEA-4 detection channel.

Click here for file

[http://www.biomedcentral.com/content/supplementary/1471213X-5-15-S2.PDF]

\section{Acknowledgements}

We would like to acknowledge Scott Noggle for magnetic bead sorting protocol; Deb Weiler for culture and maintenance of mouse embryonic fibroblast feeder layers; MaryAnne Della-Fera and Raj Rao for manuscript review and Bresagen, Inc. for supplying the hESC cells.

\section{References}

I. Thomson JA, Itskovitz-Eldor J, Shapiro SS, Waknitz MA, Swiergiel JJ, Marshall VS, Jones JM: Embryonic stem cell lines derived from human blastocysts. Science 1998, 282: I |45- I I47.

2. Shevinsky LH, Knowles BB, Damjanov I, Solter D: Monoclonal antibody to murine embryos defines a stage-specific embryonic antigen expressed on mouse embryos and human teratocarcinoma cells. Cell 1982, 30:697-705.

3. Kannagi R, Cochran NA, Ishigami F, Hakomori S, Andrews PW, Knowles BB, Solter D: Stage-specific embryonic antigens 
(SSEA-3 and -4) are epitopes of a unique globo-series ganglioside isolated from human teratocarcinoma cells. Embo J 1983, 2:2355-2361.

4. Henderson JK, Draper JS, Baillie HS, Fishel S, Thomson JA, Moore H, Andrews PW: Preimplantation human embryos and embryonic stem cells show comparable expression of stage-specific embryonic antigens. Stem Cells 2002, 20:329-337.

5. Draper JS, Pigott C, Thomson JA, Andrews PW: Surface antigens of human embryonic stem cells: changes upon differentiation in culture. J Anat 2002, 200:249-258.

6. Andrews PW, Goodfellow PN, Shevinsky LH, Bronson DL, Knowles BB: Cell-surface antigens of a clonal human embryonal carcinoma cell line: morphological and antigenic differentiation in culture. Int J Cancer 1982, 29:523-531.

7. Sotzik F, Boyd A, Shortman K: Surface antigens of human thymocyte populations defined by CD3, CD4 and CD8 expression: CDIa is expressed by mature thymocytes but not peripheral T cells. Immunol Lett 1993, 36:101-106.

8. Morrison SJ, Weissman IL: The long-term repopulating subset of hematopoietic stem cells is deterministic and isolatable by phenotype. Immunity 1994, I:66I-673.

9. Fodinger M, Fritsch G, Winkler K, Emminger W, Mitterbauer G, Gadner $\mathrm{H}$, Valent $\mathrm{P}$, Mannhalter $\mathrm{C}$ : Origin of human mast cells: development from transplanted hematopoietic stem cells after allogeneic bone marrow transplantation. Blood 1994, 84:2954-2959.

10. Hackney JA, Charbord P, Brunk BP, Stoeckert CJ, Lemischka IR, Moore KA: A molecular profile of a hematopoietic stem cell niche. Proc Natl Acad Sci U S A 2002, 99: I 306 I- 13066.

II. Palacios R, Golunski E, Samaridis J: In vitro generation of hematopoietic stem cells from an embryonic stem cell line. Proc Natl Acad Sci U S A 1995, 92:7530-7534.

12. Rao RR, Calhoun JD, Qin X, Rekaya R, Clark JK, Stice SL: Comparative transcriptional profiling of two human embryonic stem cell lines. Biotechnol Bioeng 2004, 88:273-286.

13. Richards M, Tan SP, Tan JH, Chan WK, Bongso A: The transcriptome profile of human embryonic stem cells as defined by SAGE. Stem Cells 2004, 22:5I-64.

14. Mitsui K, Tokuzawa Y, Itoh H, Segawa K, Murakami M, Takahashi K, Maruyama M, Maeda M, Yamanaka S: The homeoprotein Nanog is required for maintenance of pluripotency in mouse epiblast and ES cells. Cell 2003, I I 3:63 I-642.

15. Lalwani AK, Carey TE, Goldstein IJ, Peters BP: Lectin binding characteristics of squamous cell carcinomas of the head and neck. Acta Otolaryngol 1996, I | 6:|25-|3|.

16. Miettinen M, Holthofer H, Lehto VP, Miettinen A, Virtanen I: Ulex europaeus I lectin as a marker for tumors derived from endothelial cells. Am J Clin Pathol 1983, 79:32-36.

17. Przybylo M, Hoja-Lukowicz D, Litynska A, Laidler P: Different glycosylation of cadherins from human bladder non-malignant and cancer cell lines. Cancer Cell Int 2002, 2:6.

18. Rapoport E, Pendu JL: Glycosylation alterations of cells in late phase apoptosis from colon carcinomas. Glycobiology 1999, 9:1337-1345

19. Steck PA, Nicolson GL: Cell surface glycoproteins of I3762NF mammary adenocarcinoma clones of differing metastatic potentials. Exp Cell Res 1983, I 47:255-267.

20. Nishiyama T, Matsumoto $Y$, Watanabe H, Fujiwara M, Sato S: Detection of Tn antigen with Vicia villosa agglutinin in urinary bladder cancer: its relevance to the patient's clinical course. | Natl Cancer Inst 1987, 78: I | |3-1 I I8.

21. Arenas MI, Romo E, de Gaspar I, de Bethencourt FR, Sanchez-Chapado M, Fraile B, Paniagua R: A lectin histochemistry comparative study in human normal prostate, benign prostatic hyperplasia, and prostatic carcinoma. Glycoconj J 1999, 16:375-382.

22. Ruan S, Raj BK, Lloyd KO: Relationship of glycosyltransferases and mRNA levels to ganglioside expression in neuroblastoma and melanoma cells. J Neurochem 1999, 72:514-521.

23. Belloni PN, Nicolson GL: Differential expression of cell surface glycoproteins on various organ-derived microvascular endothelia and endothelial cell cultures. J Cell Physiol 1988, 136:398-410.

24. Takagi Y, Talbot NC, Rexroad CEJ, Pursel VG: Identification of pig primordial germ cells by immunocytochemistry and lectin binding. Mol Reprod Dev 1997, 46:567-580.
25. Brown PJ, Stephenson T]: Ulex europaeus agglutinin I lectin histochemical staining of dog hepatocellular and bile duct carcinomas. Res Vet Sci 1989, 46:42 I-423.

26. Chapman SA, Bonshek RE, Stoddart RW, Jones C], Mackenzie KR, O'Donoghue E, McLeod D: Glycoconjugates of the human trabecular meshwork: a lectin histochemical study. Histochem J 1995, 27:869-881.

27. Laitinen L, Juusela $H$, Virtanen I: Binding of the blood group-reactive lectins to human adult kidney specimens. Anat Rec 1990 , 226:10-17.

28. Goldstein IJ: Studies on the combining sites of concanavalin A. Adv Exp Med Biol 1975, 55:35-53.

29. Lotan R, Skutelsky E, Danon D, Sharon N: The purification, composition, and specificity of the anti-T lectin from peanut (Arachis hypogaea). J Biol Chem 1975, 250:85I 8-8523.

30. Goldstein IJ, Hammarstrom S, Sundblad G: Precipitation and carbohydrate-binding specificity studies on wheat germ agglutinin. Biochim Biophys Acta 1975, 405:53-61.

31. Etzler ME, Kabat EA: Purification and characterization of a lectin (plant hemagglutinin) with blood group A specificity from Dolichos biflorus. Biochemistry 1970, 9:869-877.

32. Nicolson GL, Lacorbiere M, Eckhart W: Qualitative and quantitative interactions of lectins with untreated and neuraminidase-treated normal, wild-type, and temperature-sensitive polyoma-transformed fibroblasts. Biochemistry 1975 , 14:172-179.

33. Mitalipova M, Calhoun J, Shin S, Wininger D, Schulz T, Noggle S, Venable A, Lyons I, Robins A, Stice S: Human embryonic stem cell lines derived from discarded embryos. Stem Cells 2003, 21:521-526.

34. Rosler ES, Fisk G], Ares X, Irving J, Miura T, Rao MS, Carpenter MK: Long-term culture of human embryonic stem cells in feederfree conditions. Dev Dyn 2004, 229:259-274.

35. Carpenter MK, Rosler ES, Fisk G], Brandenberger R, Ares X, Miura T, Lucero M, Rao MS: Properties of four human embryonic stem cell lines maintained in a feeder-free culture system. Dev Dyn 2004, 229:243-258.

36. Panin VM, Shao L, Lei L, Moloney DJ, Irvine KD, Haltiwanger RS: Notch ligands are substrates for protein O-fucosyltransferase-I and Fringe. J Biol Chem 2002, 277:29945-29952.

37. Lei L, Xu A, Panin VM, Irvine KD: An O-fucose site in the ligand binding domain inhibits Notch activation. Development 2003 130:64||-642|.

38. Bruder SP, Ricalton NS, Boynton RE, Connolly TJ, Jaiswal N, Zaia J, Barry FP: Mesenchymal stem cell surface antigen SB- 10 corresponds to activated leukocyte cell adhesion molecule and is involved in osteogenic differentiation. J Bone Miner Res 1998, 13:655-663.

39. Muramatsu T: Cell surface glycoproteins: biochemical, immunological and molecular biological studies. Nagoya J Med Sci 1994, 57:95-108.

40. Mason MD, Pera MF: Immunohistochemical and biochemical characterisation of the expression of a human embryonal carcinoma cell proteoglycan antigen in human germ cell tumours and other tissues. Eur J Cancer 1992, 28A: I090-1098.

4I. Cooper S, Bennett W, Andrade J, Reubinoff BE, Thomson J, Pera MF: Biochemical properties of a keratan sulphate/chondroitin sulphate proteoglycan expressed in primate pluripotent stem cells. J Anat 2002, 200:259-265.

42. Dowsing AT, Gougoulidis T, Dowsing BJ, Draber P, Trounson AO: The stage-specific expression of TEC- $1,-2,-3$, and -4 antigens on bovine preimplantation embryos. Mol Reprod Dev 1998, 49:19-28

43. Liu N, Jin C, Zhu ZM, Zhang J, Tao H, Ge C, Yang S, Zhang S: Stagespecific expression of alphal,2-fucosyltransferase and alphal, 3-fucosyltransferase (FT) during mouse embryogenesis. Eur / Biochem 1999, 265:258-263.

44. Eggens I, Fenderson B, Toyokuni T, Dean B, Stroud M, Hakomori S: Specific interaction between Lex and Lex determinants. A possible basis for cell recognition in preimplantation embryos and in embryonal carcinoma cells. J Biol Chem 1989, 264:9476-9484.

45. Taylor CT, Johnson PM: Complement-binding proteins are strongly expressed by human preimplantation blastocysts and cumulus cells as well as gametes. Mol Hum Reprod 1996, 2:52-59. 
46. Schulz TC, Palmarini GM, Noggle SA, Weiler DA, Mitalipova MM, Condie BG: Directed neuronal differentiation of human embryonic stem cells. BMC Neurosci 2003, 4:27.

47. AVarki, R Cummings, J Esko, H Freeze, G Hart, Marth J: Essentials of Glycobiology. , Cold Spring Harbor Laboratory Press; 1999:462-463.

Publish with Bio Med Central and every scientist can read your work free of charge

"BioMed Central will be the most significant development for disseminating the results of biomedical research in our lifetime. " Sir Paul Nurse, Cancer Research UK

Your research papers will be:

- available free of charge to the entire biomedical community

- peer reviewed and published immediately upon acceptance

- cited in PubMed and archived on PubMed Central

- yours - you keep the copyright

Submit your manuscript here:

http://www.biomedcentral.com/info/publishing_adv.asp
BioMedcentral 